\title{
Numerical Predictions of the Generated Work in an Air-Compression Chamber Driven by an Oscillating Water Column
}

\author{
E. G. Bautista ${ }^{1}$, F. Méndez ${ }^{*}, 2$ and O. Bautista ${ }^{1}$ \\ ${ }^{1}$ Sección de Estudios de Posgrado e Investigación, ESIME-Azcapotzalco, IPN, México, D. F., 02250, Mexico \\ ${ }^{2}$ Departamento de Termofluidos, Facultad de Ingeniería, UNAM, México, D. F., 04510, Mexico
}

\begin{abstract}
In the present work, we have studied the performance of an open compression chamber with compressed air and driven by an oscillating water column. Recognizing the existence of a free-surface for the water column, the interface position between the trapped-air and water volume -together with the motion of the column-, is described by a non-linear energy equation that reflects the main dynamic characteristics. The above governing equation is posed in dimensionless form and solved by conventional numerical methods. In addition, a theoretical approximation of the first order in $\varepsilon$ to predict the resonant frequency of the oscillatory system is derived to complete the analysis. The numerical results of the above governing equation serve us to estimate the dimensionless work done by the oscillating water column as a function of three dimensionless parameters: a characteristic Froude number, $\Gamma$, and two equivalent quasi-geometric parameters, $\beta$ and $\varepsilon$, defined-below. The predictions show that the influence of the geometry and the involved physical parameters exert a great influence on work generation into the air-chamber.
\end{abstract}

\section{INTRODUCTION}

Nowadays, the present tendency to overcome the environmental problems caused by thermal energy production is to use all available kinds of renewable energy. This enhances the status of all engineering techniques to convert the power contained in the global movement of the ocean in a renewable energy which can widely be used for a very long time. Especially the use of wave energy -a huge unexploited global power reservoir-, becomes a new centre of interest.

The Oscillating Water Column (referred to hereafter as the OWC) seen as a wave energy converter device operates mainly with conventional technologies and probably the success of these devices owes to its mechanical and structural simplicity. It consists of a partially submerged and hollow structure opened to the sea and normally, below the mean sea level. These structures partially enclose a column of water exposed to the incident wave field through the underwater opening and to the atmosphere through a power take-off mechanism. As the waves impinge on the device, pressure oscillations at the column underwater interface cause the water column rise and fall within the structure. The air trapped above the column surface within the structure is alternatively expanded and compressed through the power take-off mechanism, typically a turbine, converting the air static and dynamic pressure into mechanical energy. The principle is suitable for either bottom mounted or floating configurations. The floating device allows greater flexibility in the choice of the deployment depth and location to suit environmental and energy constraints.

* Address correspondence to this author at the Departamento de Termofluidos, Facultad de Ingeniería, UNAM, México, D. F., 04510, Mexico; Tel: +52-55-56.22.81.03; Fax: +52-55-56.22.81.06;

E-mail: fmendez@servidor.unam.mx
The conversion efficiency of an OWC may also be enhanced by beneficial interactions between the structure motion and the column oscillations. For many years, scientists and engineers have been constantly working to develop effective devices for the utilization of ocean wave-energy. An analysis of oscillating water column wave energy-device has been given by Evans [1]. He modeled the device by two closely spaced plates in two dimensions or a narrow tube of circular cross section in three dimensions. Under the assumptions of linearized water wave theory and using the method of matched asymptotic expansions, he was able to obtain the maximum power that such system can develop.

Theoretical analyses for such devices with more realistic geometries allowing spatial variations of the interior free surface were developed by Falcão and Sarmento [2] for two dimensional geometries, and later by Evans [3] who derived general results for configurations in two and three dimensions.

Long arrays of devices are required if wave energy is to provide a significant power contribution to large electrical grids. The hydrodynamic interaction between devices was first studied theoretically for systems of oscillating bodies Budal [4], Falnes and Budal, [5] and later extended to systems of oscillating pressure distribution by Evans [3]. These studies have been extended to analyze more complicated systems. For instance, Falcão [6] developed a theoretical analysis to describe the ocean wave energy absorption by a linear array of oscillating water columns of arbitrary geometry. Other fine aspects related with the operation of these devices and similar equipments were reported by GodoyDiana and Czitrom [7] to study the tuning of a seawater pump driven by a oscillating water column, and more recently, Conde and Gato [8] clarified the role of the oscillat- 


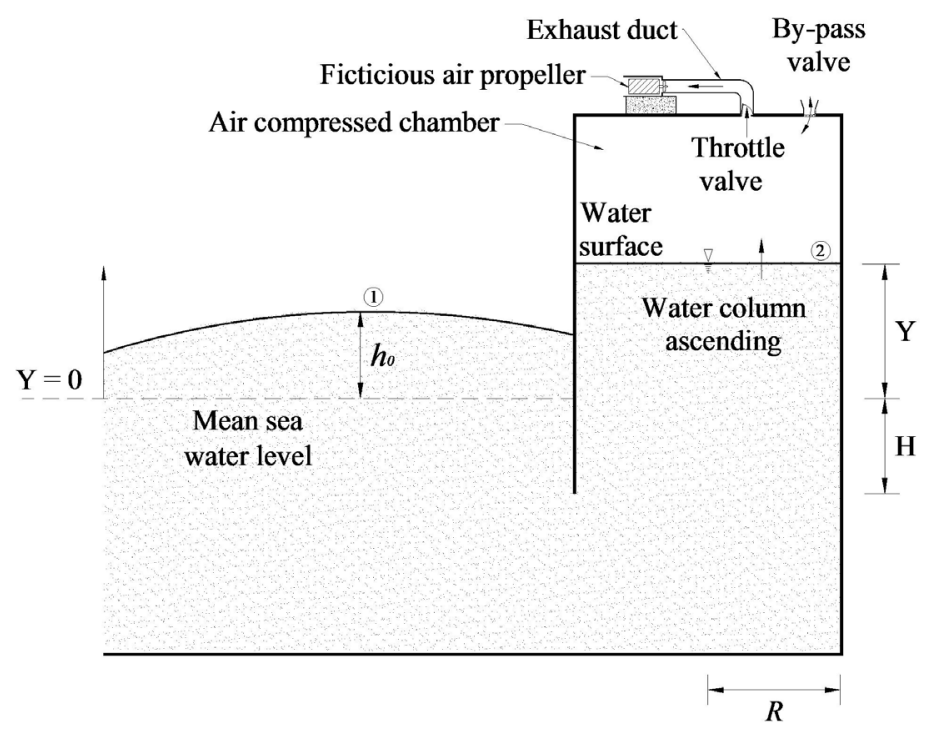

Fig. (1). Schematic diagram of the studied physical model.

ing water column on the dynamic characteristics of the airflow which is trapped inside a typical pneumatic chamber.

In this work, a theoretical analysis was developed to characterize, in a simple form, the hydrodynamics of an OWC. The oscillation characteristics of this system were described by a non-linear differential equation which predicts the amplitude of the oscillations inside the water column. In particular, the done work due to this oscillating system was evaluated as a function of the dimensionless parameters, using conventional numerical methods. In addition, we have proposed a singular perturbation method based on multiple-scales analysis, which was used in order to estimate, in a first approximation, the resonant frequency of this energy converter device. Once the amplitude of oscillation is known, the pressure and volume variations inside the air chamber were determined in order to calculate the work done.

\section{FORMULATION}

The physical model in study is shown in Fig. (1). We consider an OWC wave energy device that consists essentially of a bottom-fixed structure, whose upper part forms an air chamber and whose immersed part is open to the action of the ocean waves. In addition, the oscillating action of the waves produces a reciprocating flow of air displaced by the free surface motion. In consequence, the alternating motion of the air can drive an air turbine mounted on the top of the structure. In these systems, normally, are working a set of non-return valves to rectify the flow, considering that a conventional turbine has to be used. The details of the above system can be found elsewhere [6]. For simplicity, we adopt one dimensional formulation; therefore, it is enough to introduce a longitudinal or vertical coordinate $y$, which is measured upward in the normal direction to the mean sea water level. In the same figure, $h_{0}$ is the water wave amplitude, measured from $y=0 ; H, R$ and $Y$ represent the initial submerged length of the column, the radius of the column and the oscillation amplitude inside the water column, respectively. Because the dynamic of a OWC is generated as a con- sequence of a spreading and oscillating seawater waves, here represented by a continuous function $h(t)$; in this work for simplicity we assume the behavior of this variable is given by the following harmonic equation:

$h=h_{0} \sin \omega t$

where $\omega$ and $t$ are the frequency of the water waves and the physical time, respectively.

In order to obtain a simple mathematical model that describes the dynamics of the OWC, we consider a streamline which connects the free water surface (1) and the top of the water column (2), Fig. (1). Applying the well-known energy equation (White [9]) to the streamline from the point (1) to the point (2), we obtain

$$
\int_{1}^{2} \rho \frac{\partial V}{\partial t} d s+\left(P_{2}-P_{1}\right)+\frac{\rho}{2}\left(V_{2}^{2}-V_{1}^{2}\right)+\rho g(Y-h)+K \frac{\rho V_{2}^{2}}{2}=0
$$

where $\rho$ is the seawater density ; $P_{1}$ and $P_{2}$ are the pressures at the points 1 and 2 , respectively. In particular, physically $P_{1}$ denotes the atmospheric pressure and $P_{2}$ is the airpressure inside the chamber. $\partial V / \partial t$ represents the rate of change of the linear momentum, $g$ is the gravity acceleration, $d s$ is the arc-length along the streamline and $K$ is a global loss coefficient originated by the friction between the water column and the circular wall of the container. Furthermore, we have assumed that the viscous losses are only present inside the container. The first term of the left-hand side is approximated through the relationship,

$\int_{1}^{2} \rho \frac{\partial V}{\partial t} d s \simeq \rho\left[(Y+H) \frac{d^{2} Y}{d t^{2}}-(h+H) \frac{d^{2} h}{d t^{2}}\right]$

taking into account that $V_{2}=d Y / d t$ and $V_{1}=d h / d t$. In this form, the acceleration terms of the seawater surface level 
and the corresponding surface level inside the column are multiplied by additional apparent mass; here represented by the terms $\rho(h+H)$ and $\rho(Y+H)$, respectively. We anticipate that the influence of these factors will introduce strong non-linear effects in the final expression for the governing equation. It should be noted that this kind of simplifications have been frequently made in the past and the details can be found elsewhere, (Czitrom et al. [10]). In addition, we consider that the amplitude of the water column is sufficiently large compared with the amplitude of the water waves. Hence, we expect that $V_{2}>>V_{1}$ which reflects, implicitly, that the performance of the water column is operating in an optimal mode. The above implies that the kinetic-energy term $\rho V_{1}^{2} / 2$ is neglected in comparison with the other term $\rho V_{2}^{2} / 2$. With the aid of the above comments and simplifications, and replacing relationship (1.3) into Eq. (1.2), we obtain that,

$$
(Y+H) \frac{d^{2} Y}{d t^{2}}-(h+H) \frac{d^{2} h}{d t^{2}}+\frac{1}{\rho}\left(P_{2}-P_{1}\right)+\frac{1}{2} V_{2}^{2}+g(Y-h)+K \frac{V_{2}^{2}}{2}=0
$$

On the other hand, we adopt here the assumption of that compression process inside the air-chamber obeys the simple polytropic thermodynamic model, given by the relationship

$$
\frac{P_{2}}{P_{1}}=\left(\frac{\widetilde{V}_{1}}{\widetilde{V}_{2}}\right)^{k}
$$

where $\mathrm{k}$ is the well-known polytropic coefficient. In the above equation, we consider that the initial pressure of air inside the chamber is equal to the atmospheric pressure $P_{1}$; therefore the corresponding volume $\tilde{V}_{1}$ represents an arbitrary value of the air-volume at this pressure just before the compression process, which can easily be estimated by using the ideal-gas relationship at a standard temperature. Taking into account that, in addition, the volume $\widetilde{V}_{2}$ is a time dependent function, i. e. $\widetilde{V}_{2}=\widetilde{V}_{2}(t)$, from Fig. (1) can be appreciated this function is given by $\widetilde{V}_{2}=\widetilde{V}_{1}-\pi R^{2} Y(t)$ and denotes the variation of the air-volume inside the compression chamber as $\mathrm{Y}$ increases or decreases due to the oscillating water column. Replacing the above relationship together Eq. (1.5) into Eq. (1.4), we obtain that,

$$
\begin{aligned}
& (Y+H) \frac{d^{2} Y}{d t^{2}}-(h+H) \frac{d^{2} h}{d t^{2}}+\frac{P_{1}}{\rho}\left[\left(1-\frac{\pi R^{2} Y}{\widetilde{V}_{1}}\right)^{-k}-1\right] \\
& +\frac{1}{2}(1+K)\left(\frac{d Y}{d t}\right)^{2}+g(Y-h)=0
\end{aligned}
$$

For simplicity, Eq. (1.6) must be solved with the following initial conditions

$$
Y(t=0)=A \quad \text { and } \quad \frac{d Y(t=0)}{d t}=0
$$

where $A$ represents, in general, a characteristic amplitude of motion of the system. In this work, we choose for simplicity $A=h_{0}$.

Finally, Equation (1.6) together with the initial conditions (1.7) represents the mathematical model for this strongly non-linear oscillator. Equation (1.6) predicts the position and motion of the water column through the function $Y(t)$ and its derivative $d Y(t) / d t$ because this last represents just the velocity $V_{2}$. It should be noted that those terms of Eq. (1.6) related with the external oscillations to the cavity and represented by the function $h$ are known because this function is given by Eq. (1). Together with the assumption of a harmonic signal given by Eq. (1), we emphasize that the following hypotheses were used in deriving Eq. (1.6): one dimensional model for the energy equation with viscous losses, inclusion of apparent mass terms, a polytropic relationship to predict the pressure of the compressed air and the kinetic energy associated to the water column is larger than the corresponding kinetic energy of the motion of the seawater. In the following section, we write a more compact expression of the above equation using the dimensional analysis. In the next sections, we formulate and solve the problem using a dimensionless version of the above equations. The advantage of this formulation is that permit us to reduce the number of involved variables.

\section{NONDIMENSIONAL THEORETICAL MODEL}

A large variety of physical problems may be expressed under certain approximations and with suitable variables, in the form of one or more perturbed non-linear oscillators. We will formulate a systematic and generally applicable multiple-scale procedure for the Eq. (1.6) in order to appreciate, in a first approximation, the order of magnitude of the resonant frequency by the oscillating water column. Obviously, this parameter represents one of the most important variables under this class of problems, because the energy absorption for this converter is directly related with the resonant frequency of the system and therefore, with the performance of the system.

Introducing the following dimensionless variables,

$\tau=\omega_{N} t \quad$ and $\quad \chi=Y / h_{0}$

where $\omega_{N}$ is the natural frequency of the oscillator given by

$\omega_{N}=\left[\frac{g}{H}\left(1+\frac{{ }_{1} \pi R^{2} k P}{\rho g \widetilde{V}_{1}}\right)\right]^{1 / 2}$,

and carrying out a Taylor-expansion for the third term of the right-hand side of Eq. (1.6)

$\left(1-\frac{\pi R^{2} Y}{\widetilde{V}_{1}}\right)^{-k} \simeq 1+\left(\frac{\pi R^{2}}{\widetilde{V}_{1}}\right) k Y+\frac{1}{2}\left(\frac{\pi R^{2}}{\widetilde{V}_{1}}\right)^{2} k(1+k) Y^{2}+O\left(Y^{3}\right),(1.10)$

then, Eq. (1.6) can be transformed to 


$$
\begin{aligned}
& (Y+H) \frac{d^{2} Y}{d t^{2}}-(h+H) \frac{d^{2} h}{d t^{2}}+\frac{P_{1}}{\rho}\left(\frac{\pi R^{2}}{\widetilde{V}_{1}}\right) k Y+\frac{P_{1}}{2 \rho}\left(\frac{\pi R^{2}}{\widetilde{V}_{1}}\right)^{2} k(1+k) Y^{2}+ \\
& \frac{1}{2}(1+K)\left(\frac{d Y}{d t}\right)^{2}+g(Y-h)=0
\end{aligned}
$$

It should be noted that the natural frequency of the oscillator, given by Eq. (1.9), can be easily derived by taking the linear version of Eq. (1.11). For simplicity, the details are omitted in the present work. On the other hand, the expansion (1.10) was obtained taking into account that the ratio $\left(\pi R^{2} Y / \widetilde{V}_{1}\right) \ll 1$

\section{FORCED MOTION NEAR TO RESONANCE}

In this subsection, we analyze a fundamental aspect of all type of oscillator: the characteristic frequencies required to achieve the resonance condition of the system. In order to make it, we can introduce together with the dimensionless variables given by Equations (1.8) a small parameter $\mathcal{E}$, that basically relates the competition between no-linear and the linear effects both linked to the "spring force" of the oscillator. This parameter can be constructed by taking the ratio of the fourth term to the sum of the third and sixth term of Eq. (1.11) and considering that $Y$ reaches at least values of order $h_{0}$; in this form we have that,

$$
\varepsilon=\frac{\frac{P_{1}}{2 \rho H^{2}}\left(\frac{\pi R^{2} H}{\widetilde{V}_{1}}\right)^{2} k(1+k) h_{0}}{\left(g+\frac{P_{1} \pi R^{2}}{\rho \widetilde{V}_{1}} k\right)} \ll 1,
$$

in a similar manner, we can relate the weak driving force , $f \sim O(1)$, and the spring force with the aid of the following relationship,

$$
\varepsilon f=\frac{\text { weak driving force }}{\text { spring force }} \ll 1
$$

then

$$
\varepsilon f=\frac{(H+h) \frac{d^{2} h}{d t^{2}}}{\left(g+\frac{P_{1} \pi R^{2}}{\rho \widetilde{V}_{1}} k\right) h_{0}} \sim \frac{\left(H+h_{0}\right) \omega^{2}}{g\left(1+\frac{P_{1} \pi R^{2}}{\rho g \widetilde{V}_{1}} k\right)} \ll 1 .
$$

Near resonance, the weak driving force $f \sim O(1)$ is large enough to cause displacements of order unity and the frequency of the oscillator is near to the natural frequency. In this case, therefore, we choose $\omega / \omega_{N}=1+\varepsilon \Omega$, with
$\Omega \sim O(1)$. Thus, the driver frequency is close to resonance of the linear system.

Finally, substituting Eq. (1.1) together with the above dimensionless variables given by Eqs. $(1,8)$ and parameters, Eq. (1.11) can be written in dimensionless form as,

$$
\begin{aligned}
& (1+\beta \chi) \ddot{\chi}+\chi+\varepsilon \chi^{2}+\frac{1}{2}(1+K) \beta \dot{\chi}^{2}= \\
& \frac{g}{\omega^{2} H} \sin \left(\frac{\omega}{\omega_{N}} \tau\right)-\varepsilon f\left[1-\gamma\left(1-\sin \left(\frac{\omega}{\omega_{N}} \tau\right)\right)\right] \sin \left(\frac{\omega}{\omega_{N}} \tau\right) .
\end{aligned}
$$

Taking into account that $\omega / \omega_{N}=1+\varepsilon \Omega$; with $\Omega \sim O(1)$, Eq. (1.15) can be written in dimensionless form as

$$
\begin{aligned}
& (1+\beta \chi) \ddot{\chi}+\chi+\varepsilon \chi^{2}+\frac{1}{2}(1+K) \beta \dot{\chi}^{2}= \\
& \varepsilon f[\Gamma \sin (1+\varepsilon \Omega) \tau-\{1-\gamma(1-\sin (1+\varepsilon \Omega) \tau)\} \sin (1+\varepsilon \Omega) \tau]
\end{aligned}
$$

together with the initial conditions,

$$
\chi(\tau=0)=1 \text { and } d \chi / d \tau(\tau=0)=0 \text {, }
$$

where the dimensionless parameters are defined by

$$
\beta=\frac{h_{0}}{H}<<1 ; \gamma=\frac{h_{0}}{h_{0}+H} \quad ; \quad \Gamma=\frac{g}{\omega^{2}\left(H+h_{0}\right)},
$$

and later, in the Results section we will discuss in brevity, the physical meaning of these parameters. The convenience to write the original system in dimensionless form is that now it depends on a reduced set of dimensionless parameters and variables, $i$. $e$. the displacement of the water column is given by the function $\chi=\chi(\tau, \varepsilon f, \beta, \Gamma)$.

Equation (1.16) can be solved rigorously by using the multiple-scale analysis. However, the objective of the present paper is not to construct a formal solution with the above technique. In this context, we prefer to predict the minimal conditions to obtain a resonant frequency of this non-linear oscillator. In this context we propose the next perturbation expansion -expressed in terms of $\mathcal{E}$-, so that the resulting solution still depends, in general, on three parameters and a wide variety of phenomena can occur.

We propose the following two-scale expansion,

$$
\chi=\chi_{0}(\tau, \tilde{\tau})+\varepsilon \chi_{1}(\tau, \tilde{\tau})+\ldots
$$

where $\tilde{\tau}=\varepsilon \tau$ is a fast time scale. The details of this perturbation technique can be found elsewhere, (Kevorkian and Cole, [11]). On the other hand, we explore the asymptotic limit of $\beta \ll 1$, because the influence of this dimensionless parameter has been previously studied by Wang et al. [12].

Asymptotic Limit for $\beta<1$

In this limit, Eq. (1.16) can be readily simplified to the following 
$\ddot{\chi}+\chi+\varepsilon \chi^{2}=\varepsilon f[\Gamma \sin (\tau+\Omega \tilde{\tau})-$

$\{1-\gamma(1-\sin (\tau+\Omega \tilde{\tau}))\} \sin (\tau+\Omega \tilde{\tau})]$

Substituting Eq. (1.19) into Eq. (1.20), we obtain after collecting terms of the same power of $\mathcal{E}$, the following set of equations up to terms of order $\mathcal{E}$ :

$$
\frac{\partial^{2} \chi_{0}}{\partial \tau^{2}}+\chi_{0}=0
$$

with the initial conditions

$\chi_{0}(0,0)=a, \quad \frac{\partial \chi_{0}}{\partial t}(0,0)=0$

where $a$ is any amplitude inside the OWC.

$$
\begin{aligned}
& \frac{\partial^{2} \chi_{1}}{\partial \tau^{2}}+\chi_{1}= \\
& -2 \frac{\partial^{2} \chi_{0}}{\partial \tau \partial \tilde{\tau}}-\chi_{0}^{2}+f[\Gamma \sin (\tau+\Omega \tilde{\tau})- \\
& \{1-\gamma(1-\sin (\tau+\Omega \tilde{\tau})) \sin (\tau+\Omega \tilde{\tau})\}] .
\end{aligned}
$$

Let the solution be represented in terms of a slowly varying amplitude $R(\tilde{\tau})$ and phase $\delta(\tilde{\tau})$ relative to the driving force

$\chi_{0}(\tau, \tilde{\tau})=R(\tilde{\tau}) \cos (\tau+\Omega \tilde{\tau}-\delta(\tilde{\tau}))$

Referring to the initial conditions given by Eq. (1.22), we can choose

$R(0)=a, \quad \delta(0)=0$

Equations for the slowly varying amplitude and phase are obtained in the usual way from the condition that mixedsecular terms do not appear in the solution of $\chi_{1}$. Replacing Eq. (1.24) into Eq. (1.23), we obtain

$$
\begin{aligned}
& \frac{\partial^{2} \chi_{1}}{\partial \tau^{2}}+\chi_{1}=f[\Gamma \sin (\tau+\Omega \tilde{\tau})-\{1-\gamma(1-\sin (\tau+\Omega \tilde{\tau}))\} \sin (\tau+\Omega \tilde{\tau})]+ \\
& \sin (\tau+\Omega \tilde{\tau}-\delta(\tau)) \frac{d R(\tilde{\tau})}{d \tilde{\tau}}+R(\tilde{\tau}) \cos (\tau+\Omega \tilde{\tau}-\delta(\tau))\left(\Omega-\frac{d \delta(\tilde{\tau})}{d \tilde{\tau}}\right)- \\
& (R(\tilde{\tau}) \cos (\tau+\Omega \tilde{\tau}-\delta(\tau)))^{2}
\end{aligned}
$$

The coefficients of $\cos (\tau+\Omega \tilde{\tau}-\delta(\tau)) \quad$ and $\sin (\tau+\Omega \tilde{\tau}-\delta(\tau))$ must both vanish in Eq. (1.26) in order to avoid the secular terms. To find these equations, we use the identity

$$
\begin{aligned}
& \sin (\tau+\Omega \tilde{\tau}-\delta(\tau))=\sin (\tau+\Omega \tilde{\tau}-\delta(\tau)) \cos (\delta(\tilde{\tau})) \\
& -\sin (\tau+\Omega \tilde{\tau}-\delta(\tau)) \sin \cos (\delta(\tilde{\tau}))
\end{aligned}
$$

Then, the basic system to be studied is

$$
\frac{d R(\tilde{\tau})}{d \tilde{\tau}}+f \Gamma \cos (\delta(\tilde{\tau}))[\Gamma+\gamma-1]=0
$$

and

$$
\frac{d \delta(\tilde{\tau})}{d \tilde{\tau}}-\Omega-\frac{1}{2 R(\tilde{\tau})} f \sin (\delta(\tilde{\tau}))[1-\Gamma-\gamma]=0
$$

In the following subsections we consider two special cases to obtain some simple results.

\section{Free Un-Damped Motion}

For this case, we consider that $f=0$ and therefore, the system of Eqs. (1.27) and (1.28) reduces to

$$
\begin{array}{ll}
\frac{d R}{d \tilde{\tau}}=0, & R(0)=a, \\
\frac{d \delta}{d \tilde{\tau}}=\Omega, & \delta(0)=0 .
\end{array}
$$

The solution has $R=a=$ constant. That is, the amplitude of motion is preserved and the phase $\delta$ is

$$
\delta=\Omega \tilde{\tau} .
$$

Returning to the expansion given by Eq. (1.19) and considering Eq. (1.21), we obtain

$$
\chi=a \cos (\tau)
$$

which is a simple harmonic equation without the presence of an external force; this represents the solution for a simple oscillator with undamped motion.

\section{Forced Linear Motion}

In order to achieve this case is necessary to establish that $f=1$ and our basic system, Eqs. (1.27) and (1.28) for a slowly varying amplitude and phase becomes

$$
\begin{aligned}
& \frac{d R(\tilde{\tau})}{d \tilde{\tau}}=-\cos (\delta(\tilde{\tau}))[\Gamma+\gamma-1] \\
& \frac{d \delta(\tilde{\tau})}{d \tilde{\tau}}-\Omega=\frac{1}{2 R(\tilde{\tau})} \sin (\delta(\tilde{\tau}))[1-\Gamma-\gamma] .
\end{aligned}
$$

As $\tilde{\tau} \rightarrow \infty$, we easily obtain a steady-state behavior given by 


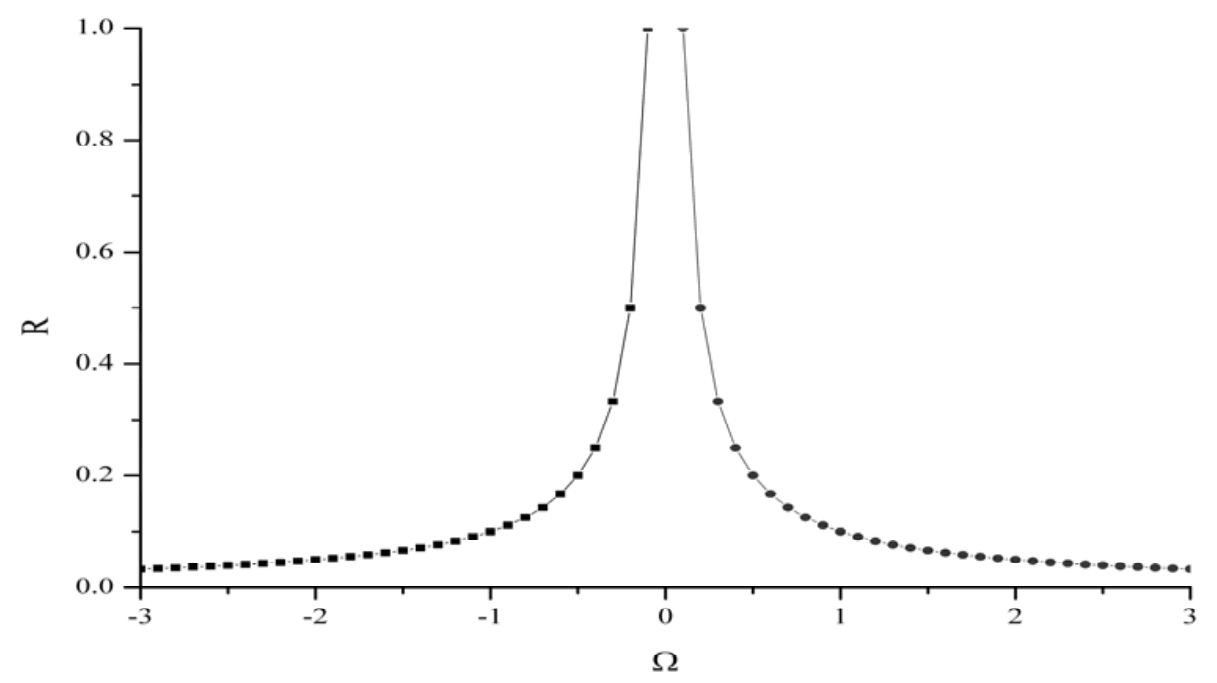

Fig. (2). Resonance curve for the limit $\mathrm{B}<<1$.

$$
\begin{aligned}
& R=\frac{1-\Gamma-\gamma}{2 \Omega} \\
& \cos \delta(\Gamma+\gamma-1)=0, \delta=\frac{\pi}{2}
\end{aligned}
$$

From equations (1.33), we can appreciate that for $|\Omega| \rightarrow \infty$ the amplitude $R \rightarrow 0$. While for $\Omega \rightarrow 0$, the amplitude has a divergent value since the first of Eqs. (1.33) gives $R \rightarrow \infty$. It can be clearly appreciated in Fig. (2). Physically, the above result means that the system is in resonance. It should be noted that under this limit $\beta \ll 1$, the damping effect represented by the fourth term of the righthand side of Eq. (1.15) was neglected. Therefore, a finite value for the amplitude does not exist. Otherwise, a complete formulation must consider the retention of the damped term of Eq. (1.15). However, in the present work the discussion for obtaining a finite value of the amplitude was not considered.

\section{NUMERICAL RESULTS}

In all numerical calculations estimated in the present work, we have used the following data $P_{1}=101.3 \mathrm{kPa}$, $\rho=1000 \mathrm{~kg} / \mathrm{m}^{3}$, and $k=1.4$ (for air). In addition, for this analysis we have chosen the geometry of the chamber with a radius $R=3 \mathrm{~m}, \widetilde{V}_{1}=300 \mathrm{~m}^{3}$ and the frequency of the external seawater wave given by $\omega=2 \mathrm{rad} / \mathrm{s}$.

The non-linear differential equation, Eq. (1.16), was solved by using the conventional Runge-Kutta method in combination with a shooting iterative scheme. We anticipate that to obtain the work done, we have used the well-know relationship,

$$
W=\int_{\widetilde{V}_{1}}^{\tilde{V}_{2}} P_{2} d \tilde{V}
$$

and combining it with Eq. (1.5) and the numerical solutions of Eq. (1.16), the results of the present section are easily derived.

In Figs. (3) and (4), we show the dimensionless amplitude $\chi$ as a function of the dimensionless time $\tau$ for some values of the involved parameters $(\Gamma=0.7, \varepsilon f=0.05$, $\varepsilon=0.04$ and 0.1 ) and two different values of $\beta=0.1$ and 0.4 , respectively. In Fig. (3), we note that for decreasing values of $\mathcal{E}$, the amplitude tends to grow up weakly, while the frequency of the oscillations remains constant for both values of $\varepsilon$. From a physical point of view, the above results have different interpretations depending on the definitions of the dimensionless parameters $\beta, \Gamma, \varepsilon f$ and $\varepsilon$. For instance, in Fig. (3) we wanted to explore the influence of the parameter $\mathcal{E}$ and those parameters that can change more easily of Eq. (1.12) are: the initial volume of the chamber $\widetilde{V}_{1}$, the initial length $h_{0}$ and the radius $R$. Therefore, for increasing values of $\varepsilon$ which means that $h_{0}$ and $R$ increase while $\widetilde{V}_{1}$ decreases, the physical amplitude tends to reach large values. Recognizing that a better operation of the OWC is to obtain higher values of the amplitude, we can conclude that the best option is to increase $R$ and to decrease $\widetilde{V}_{1}$, since both parameters can be controlled.

In a similar manner to the previous results, Fig. (4) shows the numerical solution of the nondimensional amplitude $\chi$ as a function of the dimensionless time $\tau$ for the same values of the parameters $\Gamma, \varepsilon f$ and $\varepsilon$ of Fig. (3); however, now the parameter $\beta=0.4$. Comparing with the results shown in Fig. (3), there is a small variation of the amplitude $\chi$; however, this variation is most noticeable when the air is decompressed. In this case, the influence of the parameter $\beta$, defined as the ratio of the initial amplitude of the wave to the initial submerged height of the chamber, affects obviously the displacements inside the air-chamber. From a physical point of view, again, the above result has a direct consequence: increasing values of $\beta$ means basically that the 


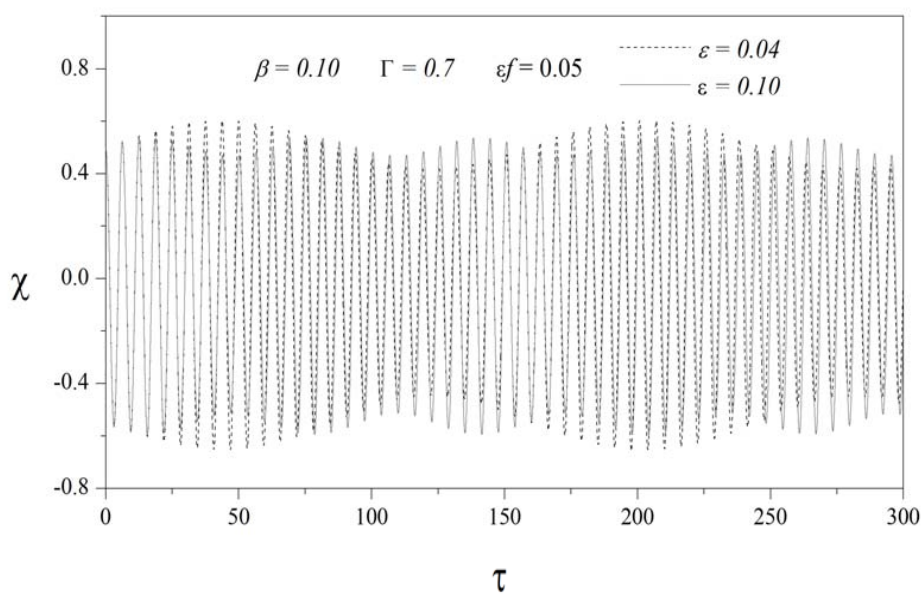

Fig. (3). Numerical solution for the nondimensional amplitude $\chi$ as a function of the dimensionless time $\tau$, for two different values of $\varepsilon$.

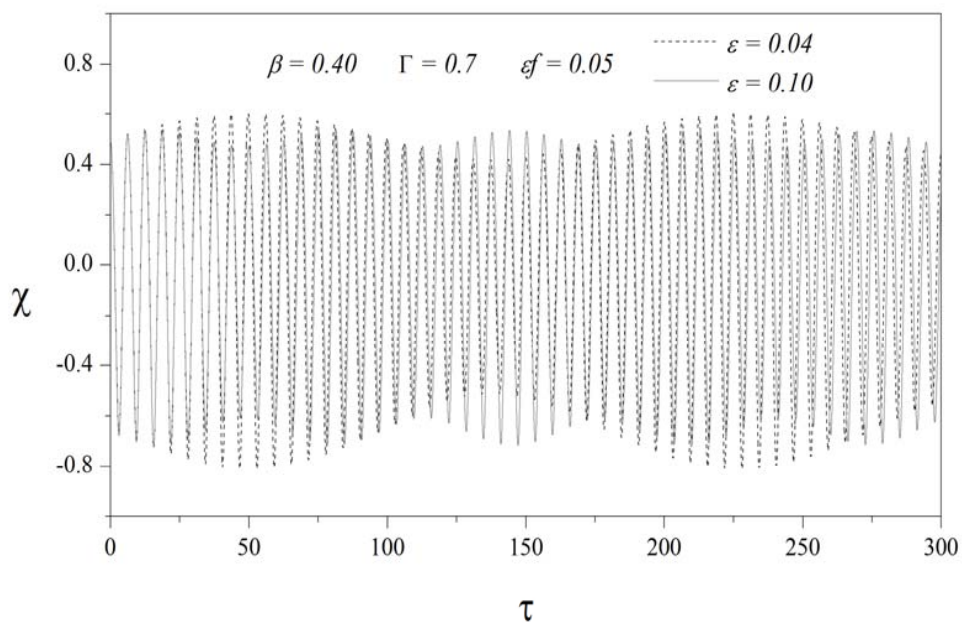

Fig. (4). Numerical solution for the nondimensional amplitude $\chi$ as a function of the dimensionless time $\tau$, for two different values of $\varepsilon$.

height $H$ of the chamber is being reduced and the physical amplitude grows weakly. Recognizing that a better operation of the OWC is to obtain higher values of the amplitude, we can conclude that the best option is to increase $H$ in order to avoid the undesirable effect of the air decompression.

The four nondimensional parameters $\beta, \Gamma, \varepsilon f$ and $\varepsilon$ show the influence of different physical variables basically related with geometrical lengths, spring and external forces. The parameter $\Gamma$ that defines the characteristic Froude number for this problem, involves to the gravity constant $g$, the external frequency $\omega$, the initial amplitude $h_{0}$ and the submerged length of the column $H$. Obviously, the Froude number has a great influence on the hydrodynamics oscillation, situation that can be seen in Fig. (5). In this figure, the numerical predictions for the nondimensional amplitude $\chi$ as a function of the time, $\tau$, are shown by imposing a value of $\Gamma=1.4$ and the same values of the parameters $\beta, \varepsilon f$ and $\varepsilon$ used in Fig. (3). Comparing the results of Fig. (3) against the results of Fig. (5), we can appreciate that the amplitude $\chi$ has the higher values of the amplitude for this last figure, reaching practically twice the value of $h_{0}$. Between both figures, from a physical point of view, we can conclude that for smaller values of the physical frequency $\omega$ the amplitude grows and therefore, a better operation of the OWC device is achieved under this condition.

In Fig. (6), the numerical solution for the nondimensional amplitude $\chi$ versus the nondimensional time, $\tau$, is shown by imposing a value of $\Gamma=1.4$ and the same values of the parameters $\beta, \varepsilon f$ and $\varepsilon$ used in Fig. (4). Comparing the results of Fig. (4) against the results of Fig. (6), we appreciate that the amplitude $\chi$ has higher values of the amplitude for the last figure. From a physical point of view, we can conclude, again, that for smaller values of the physical frequency $\omega$ the physical amplitude grows; however, the decompression condition into the chamber is now stronger. Therefore, the optimal election is the case treated in Fig. (4), where the decompression effects are diminished.

Fig. (7) shows the nondimensional work as a function of the parameter $\varepsilon$ and a fixed value for the Froude number, $\Gamma=0.7$. In this figure, we can appreciate that in the limit 


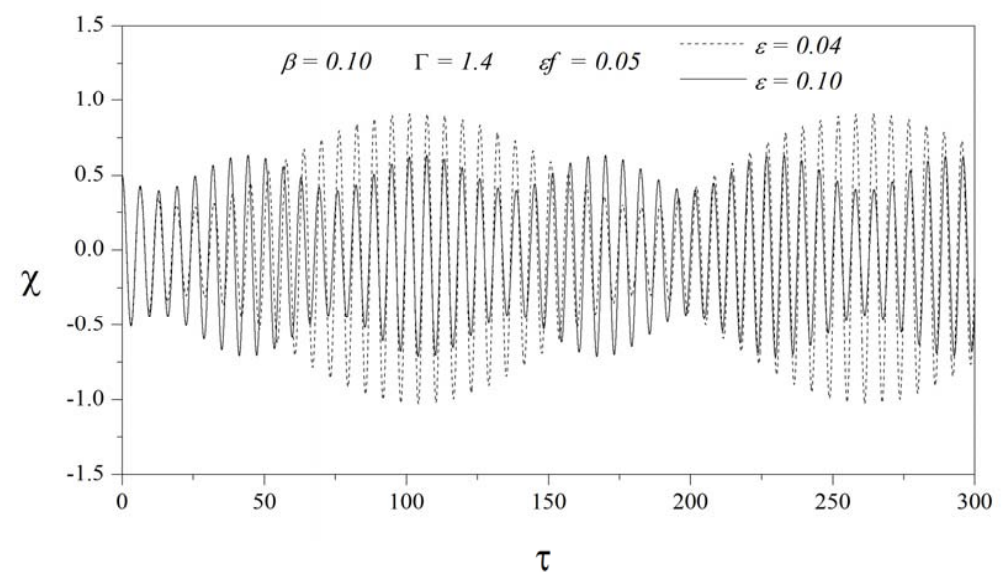

Fig. (5). Numerical solution for the nondimensional amplitude $\chi$ as a function of the nondimensional coordinate $\tau$, for two different values of $\varepsilon$.

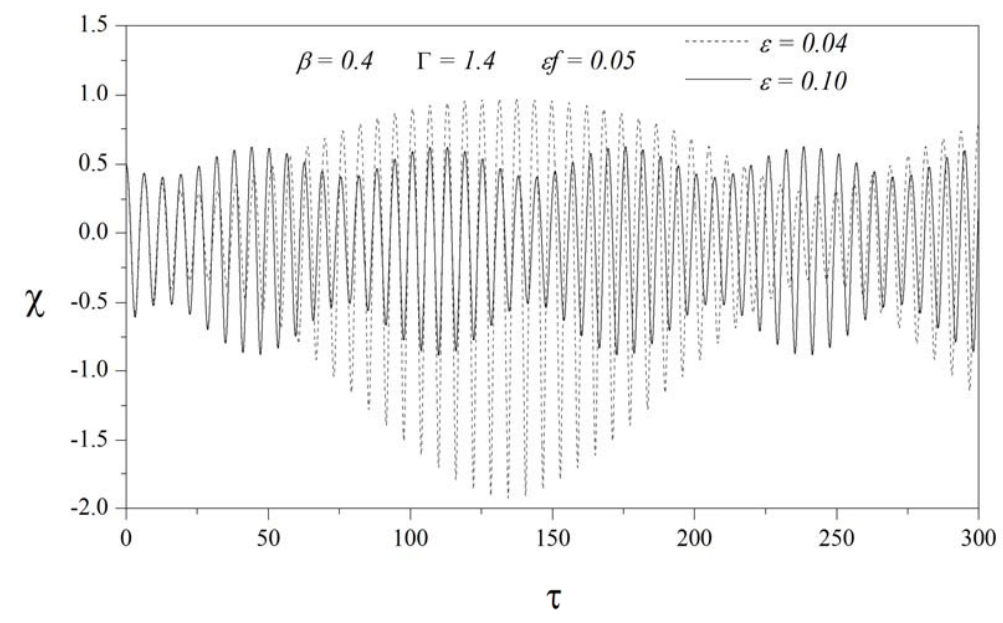

Fig. (6). Numerical solution for the nondimensional amplitude $\chi$ as a function of the dimensionless time $\tau$, for two different values of $\varepsilon$.

of $\varepsilon \rightarrow 0$, the work reaches maximum values of $4.8 \times 10^{-3}$ and $5.6 \times 10^{-3}$ for the values of the parameter $\beta=0.32$ and $\beta=0.16$, respectively. From a physical point of view, the above result means that is more effective to increase the initial submerged length of the column since in this form the work done is higher. However, it depends on the assumed values of the initial volume $\widetilde{V}_{1}$ and the radius $R$. For example, an adequate operation of the OWC device can be reached with small values $\widetilde{V}_{1}$ and large values of $R$. This is a direct consequence of the definition of the parameter $\mathcal{E}$. In addition, an oscillating behavior for the work done is also obtained. The physical explanation is due to that the decompression effects play a significant role to control the corresponding oscillations. It was previously discussed in Figs. (4) and (6).

Finally, the work done as a function of the dimensionless parameter $\mathcal{E}$ under similar conditions of those shown in Fig. (7), except the Froude number produces practically a unique maximum value of the work done of order of $9.0 \times 10^{-3}$ for $\beta=0.32$ and $\beta=0.16$. In Fig. (8), the value of Froude number has been increased twice and the result is to increase even more the work done. From a physical point of view, we clarify for this figure the influence of the frequency $\omega$. For smaller values of the ocean wave frequency, the motion of the column absorbs more energy and therefore is easier to obtain the largest amounts of work done by the water column.

\section{DISCUSSION AND CONCLUSIONS}

In the present work, a very simple model based on the energy equation for predicting the non-linear oscillations of a water column that is pushing a fixed volume of trapped air is analyzed. The main objective was to predict the work done inside the air-chamber. Obviously, the resonance condition must be determined as a part of the problem since the optimal operation of these energy absorbing devices are regulated by this condition. In order to make it easier and recognizing that the number of physical variables and properties are large, we have derived and solved the energy equation in a dimensionless form in order to reduce the involved parameters. In this manner, the motion of the water column and the work done depend basically on four dimensionless pa- 


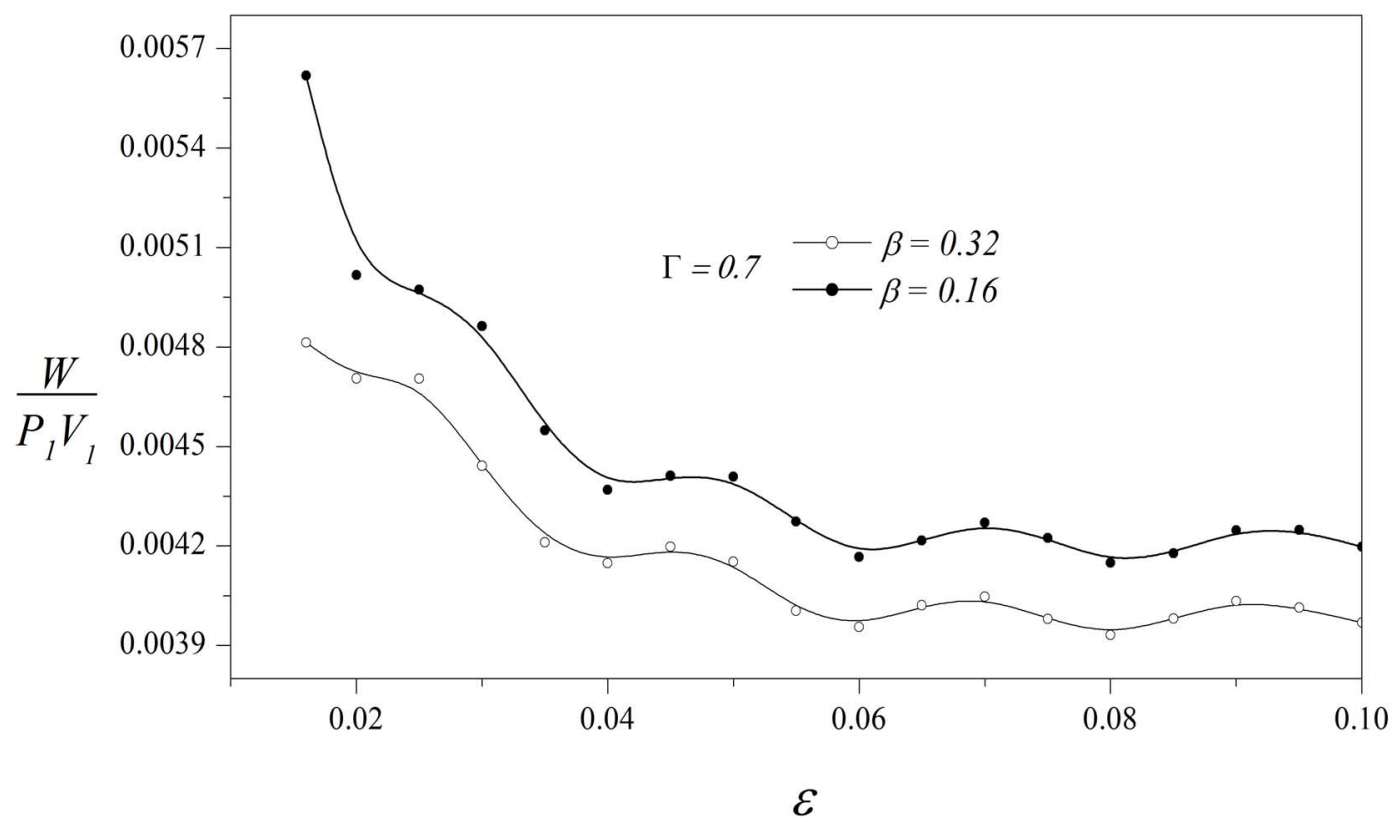

Fig. (7). Nondimensional work as a function of the parameter $\mathcal{E}$ for two different values of $\beta$.

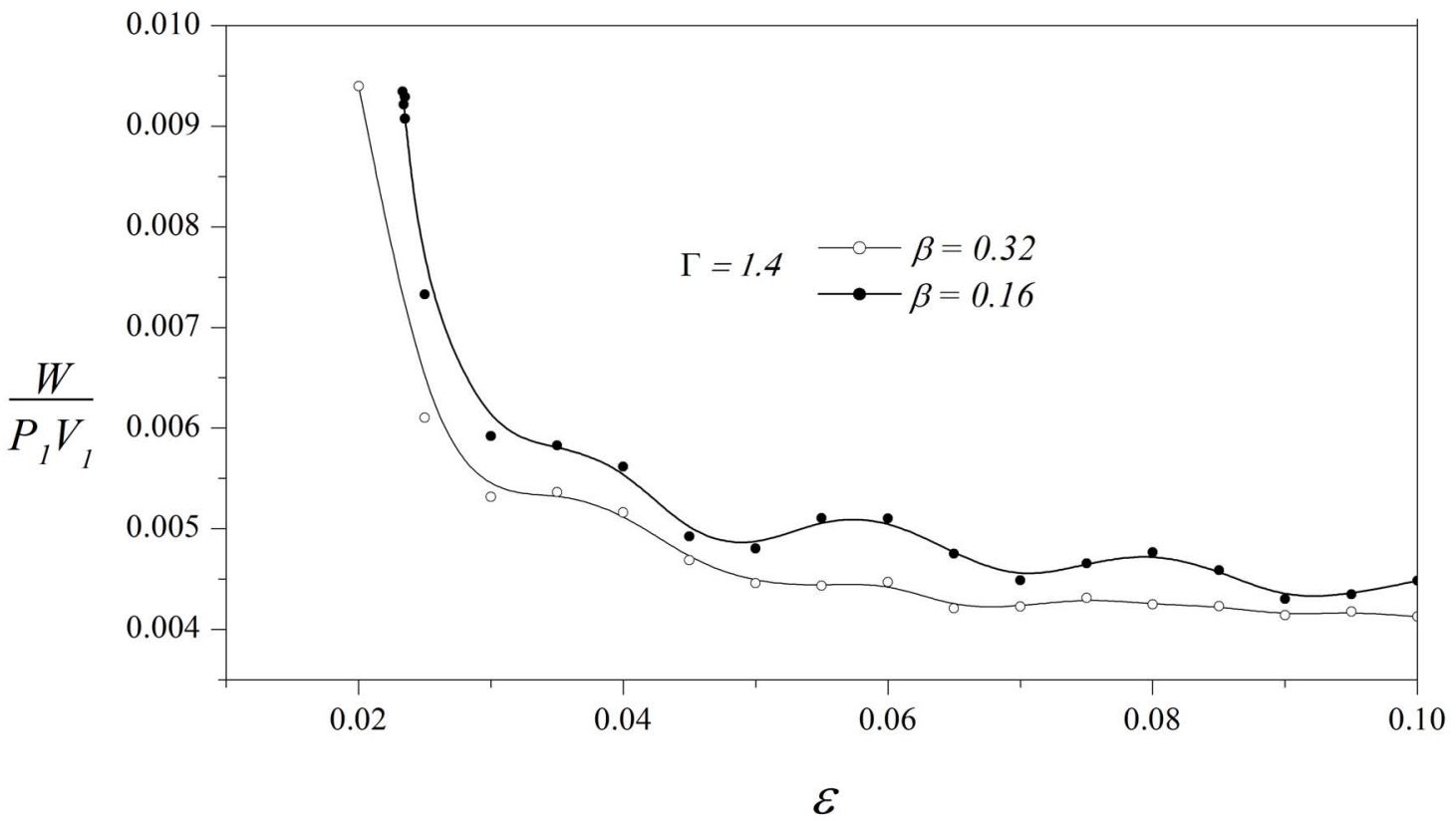

Fig. (8). Nondimensional work as a function of the parameter $\varepsilon$ for two different values of $\beta$.

rameters: $\beta, \Gamma, \varepsilon f$ and $\varepsilon$. The physical influence of these dimensionless parameters was widely discussed and serves us to characterize the basic operation of this energy converter device. In particular, we have shown that the hydrodynamic of an Oscillating Water Column (OWC) is strongly influenced by the oscillation conditions of the ocean waves, controlled mainly by the frequency and the amplitude of the ocean waves. One limitation of the analysis is that the parameter $\beta$ must be small in order to guarantee a continuity condition through the seawater-OWC system; particularly, it does not have to be greater than $1 / 2$. Clearly, the numerical solutions show that the maximum amplitude of the water column inside the structure occurs for small values of $\mathcal{E}$. The above results are in concordance with the predictions of Figs. (7) and (8), since the maximum values of the work done satisfy also this condition. In those figures, when $\Gamma$ increases, the ocean wave frequency $\omega$ decreases and for this case, we have the maximum work generated by the compressed air. Recognizing that the definition of the efficiency of the energy convert device is, in general an arbitrary condition, we postulate that the dimensionless version of the work done represents just the efficiency. Therefore, both figures are maps for the performance of these devices. 


\section{ACKNOWLEDGEMENT}

E. G. Bautista thanks to DGAPA, UNAM for the received support during a post-doctoral scholarship.

\section{REFERENCES}

[1] D. E. Evans, "The oscillating water column wave-energy device," J. Inst. Math. Appl., vol. 22, pp. 423-433, December 1978.

[2] A. F. de O. Falcão and A. J. N. A. Sarmento, "Wave generation by a periodic surface pressure and its application in wave-energy extraction," in 15th International Congress of Theoretical and Applied Mechanics, Toronto, August 1980.

[3] D. E. Evans, "Wave power absorption by systems of oscillating surface-pressure distributions", J. Fluid Mech., vol. 114, pp. 481499, April 1982.

[4] K. Budal, "Theory for absorption of wave power by a system of interacting bodies," J. Ship Res., vol. 21, pp. 248-253, September 1977.

[5] J. Falnes, K. Budal, "Wave-power conversion by point absorbers," Norwegian Marit. Res., vol. 6, pp. 2-11, June 1978.
[6] A. F. de O. Falcão, "Wave-power absorption by a periodic linear array of oscillating water columns," Ocean Eng., vol. 29, pp. 1163 1186, August 2002.

[7] R. Godoy-Diana, and S. P. R. Czitrom, "On the tuning of a waveenergy driven oscillating-water-column seawater pump to polychromatic waves," Ocean Eng., vol. 34, pp. 2374-2384, December 2007.

[8] J. M. Paixão Conde, and L. M. C. Gato, "Numerical study of the air-flow in an oscillating water column wave energy converter," Renew. Energy, vol. 33, pp. 2637-2644, December 2008.

[9] F. M. White, Fluid Mechanics, 5th ed. New York: McGraw-Hill Science, 2003

[10] S. Czitrom, R. Godoy, E. Prado, P. Pérez, and R. Peralta-Fabi, "Hydrodynamics of an oscillating water column seawater pump. Part I: theoretical aspects," Ocean Eng., vol. 27, pp. 1181-1198, November 2000

[11] J.K. Kevorkian and J.D. Cole, Multiple Scale and Singular Perturbation Methods. New York: Springer-Verlag, 1996.

[12] D. J. Wang, M. Katory, and Y. S. Li, "Analytical and experimental investigation on the hydrodynamic performance," Ocean Eng., vol. 29, pp. 871-885, July 2002.

(C) Bautista et al.; Licensee Bentham Open.

This is an open access article licensed under the terms of the Creative Commons Attribution Non-Commercial License (http://creativecommons.org/licenses/by-nc/3.0/) which permits unrestricted, non-commercial use, distribution and reproduction in any medium, provided the work is properly cited. 